doi:10.12662/2359-618xregea.v10i2.p80-99.2021

ARTIGO

\section{DIRETRIZES PARA CONSTRUÇÃO DE TRILHAS DE APRENDIZAGEM PARA DESENVOLVIMENTO DE PROFISSIONAIS DO SETOR INDUSTRIAL DA SOCIEDADE EM TRANSFORMAÇÃO DIGITAL}

\section{GUIDELINES FOR BUILDING LEARNING TRAILS FOR DEVELOPING PROFESSIONALS FROM THE INDUSTRIAL SECTOR OF THE SOCIETY IN DIGITAL TRANSFORMATION}

\section{RESUMO}

A Sociedade em Transformação Digital tem exigido um conjunto de novas competências dos profissionais de diversos setores, estimulando as organizações a buscarem novos meios de proporcionar a seus profissionais o desenvolvimento contínuo que, alinhado com seus objetivos organizacionais, possibilita a construção de valores distintivos em prol do desenvolvimento corporativo. Como facilitar o desenvolvimento de novas competências ao longo da vida profissional é um tema frequentemente abordado em pesquisas acadêmicas e buscado pelas organizações no mundo corporativo. Uma estratégia de gestão do desenvolvimento humano nas organizações se baseia na elaboração de Trilhas de Aprendizagem. Porém, há lacuna de conhecimento científico sobre as diretrizes que devem ser consideradas para o desenvolvimento de Trilhas de Aprendizagem, especificamente para a Sociedade em Transformação Digital. Com o objetivo de identificar diretrizes para construção de Trilhas de Aprendizagem para desenvolvimento de competências nos profissionais do setor industrial para a Sociedade em Transformação Digital, foi realizada uma revisão sistemática da literatura em bases de dados internacionais, que, somada a uma análise documental e bibliográfica exploratória, resultou na proposição de uma série de diretrizes a serem consideradas na construção dessas Trilhas de Aprendizagem.

Palavras-chave: Sociedade em Transformação Digital. Indústria 4.0. Desenvolvimento de Competências. Competências. Trilha de Aprendizagem.

\footnotetext{
ABSTRACT

The Society in Digital Transformation has required a set of new skills from professionals from different sectors, encouraging or-
} 
ganizations to seek new ways to provide their professionals with continuous development that, in line with their organizational goals, enable the construction of distinctive values in favor of corporate development. How to facilitate the development of new skills throughout professional life is a topic frequently addressed in academic research and pursued by organizations in the corporate world. A human development management strategy in organizations is based on the elaboration of Learning Paths. However, there is a lack of scientific knowledge about the guidelines that should be considered for the development of Learning Paths, specifically for Society in Digital Transformation. In order to identify guidelines for the construction of Learning Paths for the development of skills in the industrial sector professionals for the Society in Digital Transformation, a systematic literature review was carried out in international databases, which added to an exploratory documentary and bibliographic analysis, resulted in the proposition of a series of guidelines to be considered in the construction of these Learning Paths.

Palavras-chave: Society in Digital Transformation. Industry 4.0. Skills Development. Skills. Learning Trail.

\section{INTRODUÇÃO}

A Sociedade em Transformação Digital (STD), da mesma forma que as outras revoluções vividas pela humanidade, tem exigido profissionais com novas competências, tornando o desenvolvimento dos trabalhadores um dos principais desafios das organizações, junto com compartilhamento de conhecimento e implementação de novas tecnologias (AIRES; KEMPNER-MOREIRA; FREIRE, 2017; AIRES, 2020).

O termo Sociedade em Transformação Digital (STD) também é encontrado na literatura como Indústria 4.0 (SCHWAB, 2016; CONFEDERAÇÃO NACIONAL DA INDÚSTRIA, 2016; STEVAN JÚNIOR; LEME; SANTOS,
2018), Manufatura Avançada ou Indústrias Inteligentes (CONFEDERAÇÃO NACIONAL DA INDÚSTRIA, 2016), Fábricas inteligentes (COSTA; STEFANO, 2014; SCHWAB, 2016), Transformação Digital (SCHWAB, 2016; MCKINSEY, 2016) ou Quarta Revolução Industrial (SCHWAB, 2016; STEVAN JÚNIOR; LEME; SANTOS, 2018).

Neste estudo, optou-se por adotar o termo Sociedade em Transformação Digital (STD) por entender que melhor descreve o período que a humanidade está experienciando, não se limitando apenas ao setor industrial, mas a todos os setores econômicos e à vida privada dos indivíduos.

A forma de desenvolver as competências individuais dos trabalhadores foi sendo aprimorada com o passar dos anos, acompanhando as necessidades de cada período. Na Antiguidade, a escala de produção era baixa e, geralmente, os bens eram produzidos para o próprio consumo (SLEIGHT, 1993; HARARI, 2018). Antes do ensino formal, o desenvolvimento das competências profissionais, muitas vezes, acontecia no seio familiar, ensinadas de pai para filho, sendo comum os filhos seguirem a mesma atividade laboral que seus pais (CORDÃO; MORAES, 2017; HARARI, 2018).

Em uma perspectiva histórica do processo evolutivo da espécie humana, conforme os anos passaram, fomos deixando de ser generalistas e tornando-nos especialistas, cada vez mais dependendo das competências uns dos outros para sobrevivermos (HARARI, 2018).

Assim, acompanhando o desenvolvimento tecnológico de cada revolução vivida pela humanidade, especialmente das revoluções industriais, a relação de competências exigidas dos profissionais também foi-se atualizando, dando espaço para o surgimento da educação corporativa (AIRES; FREIRE; SOUZA, 2016), que surgiu justamente com o objetivo de reduzir lacunas de desenvolvimento de competências, sendo estas por falhas na formação acadêmica/escolar ou pela necessidade de requalificação dos profissionais (EBOLI, 2004; MEISTER, 1999; FREIRE et al., 2016a). 
Por sua vez, a educação corporativa também foi evoluindo, indo além dos programas de educação de funcionários, criando sistemas de aprendizagem contínua vinculadas às suas estratégias e metas envolvendo funcionários, clientes e cadeia de fornecimento (MEISTER, 1999), dando espaço para o desenvolvimento de diversos modelos de Educação Corporativa (FREIRE et al., 2016b), bem como estratégias de desenvolvimento ágil das competências desejadas.

Contudo, a educação formal não ficou parada, houve esforços para garantir o alinhamento dos currículos escolares com as demandas do mercado de trabalho, prevendo esta necessidade na legislação educacional (CORDÃO; MORAES, 2017).

Na educação formal brasileira, o modelo de desenvolvimento de competências surgiu por meio das reformas educacionais realizadas na década de 1990, que foram alinhadas com as reformas de ajuste macroeconômico, realizadas no mesmo período em que buscava superar a inflação e a estagnação, retomando o crescimento econômico dos anos 1980, conhecida como a década perdida (DELUIZ, 2001).

Em 1996, essa tentativa de aproximação do mundo escolar com o mundo do trabalho pode ser observada na Lei $n^{0} 9.394$, de 20 de dezembro de 1996, que estabeleceu as diretrizes e bases da educação nacional no Brasil, conhecida no dia a dia escolar pela sigla: LDB, que apresenta, logo em seu início, a relação da educação formal com o mundo do trabalho ao mencionar, em seu artigo primeiro, que: " $\$ 2^{\circ}$ A educação escolar deverá vincular-se ao mundo do trabalho e à prática social. " (BRASIL, 1996, p. 1).

Em 2000, esse esforço de atualizar os currículos da educação formal com as necessidades do mundo do trabalho pôde ser observado nos Referenciais Curriculares Nacionais da Educação Profissional de Nível Técnico (RCNEP), publicado pelo Ministério da Educação, que trouxe a abordagem metodológica de desenvolvimento de competência e a necessidade de reposicionamento dos currículos, listando as competências profissionais gerais do profissio- nal técnico de vinte áreas tecnológicas. Além disso, apontou a necessidade do desenvolvimento de planos de cursos personalizados definindo as competências e as habilidades a serem desenvolvidas e as bases tecnológicas necessárias (BRASIL, 2000).

Mais tarde, em 2018, na última revisão das diretrizes curriculares nacionais do Ensino Médio (BRASIL, 2018), valorizou a formação profissional, apresentando Competências Gerais alinhadas com o mundo do trabalho e tendo um dos eixos formativos a Educação Profissional.

Desse modo, no que tange aos aspectos legais educacionais brasileiros, está declarado que as competências para o mundo do trabalho devem ser construídas desde a escola, conforme defendido por Perrenoud (1999).

Sobre a Educação Profissional, historicamente no Brasil, esse termo remete à formação da classe operária, sendo o ensino superior reservado para a elite; contudo, Cordão e de Moraes (2017) apresentam uma análise contemporânea afirmando que "depois da educação básica, tudo é educação profissional," (CORDÃO; MORAES, 2017, p. 77). Na visão dos autores, todos os cursos formais ofertados após a conclusão do ensino médio é educação profissional, desde cursos técnicos, passando pela graduação, sequenciais e pós-graduação (lato sensu e stricto sensu), o que muda em cada nível de ensino é "o grau de sofisticação dos conteúdos estudados e as competências esperadas no perfil de conclusão, com variação correspondente na carga horária e anos de duração." (CORDÃO; MORAES, 2017, p. 77). Assim, se para ser cientista a formação necessária é doutorado, esta é a educação profissional necessária para atuar nesta ocupação laboral.

Trazendo para o mercado de trabalho, por que desenvolver competências desde a escola é importante? Porque indivíduos com um conjunto de competências desenvolvidas são capazes de se antecipar a situações, sabem agir em situações de incerteza e são capazes de mobilizar múltiplos conhecimentos, ou seja, sabem "entender, antecipar, avaliar e enfren- 
tar a realidade com ferramentas atuais" (PERRENOUND, 1999, p. 11), serão profissionais completos e preparados para enfrentar o mundo do trabalho. Afinal, para que serviria aprender um arcabouço de conhecimentos teóricos se não for capaz de o transferir para sua prática do trabalho?

Neste sentido, diferente dos recursos tradicionais, conhecimento não pode ser comprado, precisa ser desenvolvido. "Uma das estratégias utilizadas para renovar e aumentar o estoque de conhecimento organizacional é o investimento em capacitação de pessoal." (STEIL, 2006, p. 6). E as organizações investem em treinamento para seus funcionários com o intuito de aumentarem sua produtividade e competitividade, logo, aumentar o seu lucro (STEIL, 2006).

Trazendo a discussão para a Sociedade em Transformação Digital, são inúmeros os estudos científicos e empíricos que apresentam listas de competências a serem desenvolvidas pelos profissionais, muitas delas relacionadas às tecnologias desta Revolução Industrial; contudo, no meio científico, carecem de estudos que tragam como desenvolver tais competências, que apresentem diretrizes para a construção de Trilhas de Aprendizagem de competências para a Sociedade em Transformação Digital (AIRES, 2020).

Nesse contexto de emergência de desenvolvimento de novas competências nos profissionais da Sociedade em Transformação Digital, surge o objetivo deste estudo: identificar diretrizes para a construção de Trilhas de Aprendizagem para desenvolvimento de competências nos profissionais do setor industrial para a Sociedade em Transformação Digital.

Tais diretrizes são importantes para ilustrar o passo de como construir uma oferta formativa no formato e Trilha de Aprendizagem reunindo o conjunto de competências listadas que precisam ser desenvolvidas pelos profissionais por meio de suas lacunas de competências individuais alinhadas com os objetivos organizacionais.

\section{REVISÃO DA LITERATURA}

O primeiro passo para desenvolver competências é mapear qual o conjunto de competências que os profissionais precisam ter com base nos objetivos organizacionais; o segundo passo é identificar as lacunas de competências dos profissionais contratados, para, então, chegar ao terceiro passo: listar quais as competências que precisam ser priorizadas nas formações ofertadas à força de trabalho. Identificar esse gap de competências é fator crítico no processo de desenvolvimento dos profissionais e, consequentemente, desenvolvimento organizacional (GUIMARÃES et al., 2001; BRANDÃO, 2017).

Em uma visão contemporânea, espera-se que a relação de competências a serem desenvolvidas transcenda a relação de competências técnicas relacionadas às tecnologias 'usadas na atividade laboral, valorizando, também, as competências socioemocionais (SENAI, 2019; FREIRE et al., 2019), as competências STEAM e as competências gerenciais (FREIRE et al., 2019).

No mercado competitivo, em que o conhecimento dos trabalhadores é fator crítico de sucesso e precisa ser considerado como recurso estratégico das organizações (DAVENPORT; PRUSAK, 1998; SENGE, 2012; SCHWAB, 2016; CONFEDERAÇÃO NACIONAL DA INDÚSTRIA, 2016; SCHWAB; DAVIS, 2018), as empresas estão, constantemente, desafiadas a facilitar a aprendizagem de seus funcionários, criando estratégias de que o desenvolvimento de novas competências seja constante (FREITAS; BRANDÃO, 2006; ALLES, 2007).

Neste sentido, Le Boterf(1999) lista que, para desenvolver competência, são necessários três fatores básicos: 1) indivíduo que queira aprender, 2) incentivo para o aprendizado, 3) ofertas formativas disponíveis. Zarifian (1996) acrescenta dizendo que as empresas não podem apenas ser qualificadas, mas "qualificantes", ou seja, precisam ofertar constantemente possibilidades de formação para seus trabalhadores.

Le Boterf (1999), Freitas (2002) e Antonello (2007) afirmam que a formação de compe- 
tências vai além de grades de formação prescritivas, apontando que aprender vai muito além de currículos pré-determinados. Aprender também ocorre no ambiente de trabalho em atividades não estruturadas, mas não sem objetivo (ALLES, 2007; ANTONELLO; FLACH, 2011).

Nesta conjuntura de demanda constante por formação dos profissionais, as grades de treinamento têm sido criticadas por levarem em consideração, muitas vezes, apenas a estrutura de cargos da empresa, inibindo o desenvolvimento de competências que vão além do prescritivo dos cargos (FREITAS; BRANDÃO, 2006; BRANDÃO, 2017), não considerando que as estruturas das empresas evoluem, sendo cada vez menos estáveis e lineares (GUI, 2000). A premissa básica do treinamento para formação de competências é o alinhamento com as diretrizes estratégicas organizacionais (JUNQUEIRA, 2000) e não com a descrição de cargos (FREITAS; BRANDÃO, 2006; BRANDÃO, 2017).

Neste cenário, surgem as Trilhas de Aprendizagem como forma de facilitar o desenvolvimento profissional. As trilhas de aprendizagem foram desenvolvidas no contexto da "gestão baseada por competências" com o objetivo de sustentar e desenvolver competências nos trabalhadores alinhadas com os objetivos organizacionais das empresas, buscando, além da competitividade organizacional, o desenvolvimento integral de seus trabalhadores, indo além de desempenhos superiores na empresa, mas buscando desenvolver suas aspirações pessoais também (FREITAS; BRANDÃO, 2006; ALMEIDA, 2013; BRANDÃO, 2017).

Trilhas de Aprendizagem são caminhos alternativos para o desenvolvimento profissional, são flexíveis e não prescritivos (FREITAS, 2002), conectando iniciativas formais e informais (ANTONELLO; FLACH, 2011). O termo learning (aprendizagem em inglês) significa trilha ou sulco na terra, em referência à atividade de preparar o solo para o plantio (SENGE, 1998). Trazendo essa analogia para o mundo do trabalho, aprender uma nova competência é preparar o profissional para novos resultados; na plantação, esse resultado é colheita. Aprender, portanto, pode ser entendido como o ato de buscar elevar o nível de competências por meio de trilhas de aprendizagem (JUNQUEIRA, 2000).

Um profissional, ao escolher o próximo curso que irá realizar, deve analisar suas competências atuais, suas aspirações futuras, sua forma de aprendizagem preferida, as ofertas formativas disponíveis, os recursos educacionais disponíveis e outras variáveis para, então, definir sua próxima etapa educacional (FREITAS; BRANDÃO, 2006; BRANDÃO, 2017). Por isso, é importante serem consideradas no desenvolvimento de Trilhas de Aprendizagem questões relacionadas ao perfil dos profissionais a serem desenvolvidos, como forma de aprendizado preferida e outros aspectos pertinentes que traduzam quem é o público-alvo que será desenvolvido (ALVES, 2016), possibilitando, assim, que ajustes e personalizações sejam realizadas conforme o grupo a ser capacitado.

Da mesma forma, as organizações avaliam sua estratégia, as competências de seus profissionais, a possibilidade de contratar competências externas e outras variáveis para, então, decidir quais treinamentos serão oferecidos a seus funcionários, visando ao alcance de seus objetivos organizacionais. Ambos, tanto o profissional, quanto a organização, buscam a mesma coisa, um desempenho superior no futuro comparado ao que já tenha conquistado no presente (FREITAS; BRANDÃO, 2006; BRANDÃO, 2017).

A obrigatoriedade de participação de uma grade de treinamentos sem considerar as competências já desenvolvidas e experiências anteriores da força de trabalho é desperdício de recursos, para a empresa, e pouco atraente do ponto de vista do empregado (FREITAS, 2002; ANTONELLO, 2011). A possibilidade de o empregado escolher quais cursos quer fazer entre uma seleção disponível vai muito além de sua participação na escolha de seu desenvolvimento profissional, cultiva uma harmonia entre interesses pessoais e da organização (LE BOTERF, 1999). Desenvolver competências 
não pode ser uma ação forçada, é dar a oportunidade de as pessoas querem mudar seu EU de amanhã (JUNQUEIRA, 2000).

As Trilhas de Aprendizagem diferem de grades de treinamento por serem flexíveis ao ponto de oferecer uma formação personalizada para cada indivíduo (FREITAS; BRANDÃO; 2006; ALMEIDA, 2013). Cada profissional pode seguir uma trilha diferente do outro usando diferentes estratégias de ensino e aprendizagem. Nas Trilhas de Aprendizagem, podem ser realizadas diversas estratégias de aprendizagem: treinamentos autoinstrucionais, cursos presenciais, cursos a distância, estágios, viagens de estudo, seminários, palestras, leitura de revistas, livros, jornais, grupos de estudo e diversos outros meios de aprendizagem (FREITAS; BRANDÃO; 2006; ANTONELLO; FLACH, 2011; ALMEIDA, 2013; BRANDÃO, 2017).

Neste contexto, um "profissional A" e um "profissional B" podem desenvolver a mesma competência desejada, mas percorrendo Trilhas de Aprendizagem diferentes (LE BORTEF, 1999). As Trilhas de Aprendizagem podem ser individualizadas, respeitando o ritmo, as preferências e as limitações de aprendizado de cada indivíduo (FREITAS, 2002; ALMEIDA, 2013).

Ao adotar Trilhas de Aprendizagem, as organizações possibilitam ao trabalhador o desenvolvimento de competências de forma não prescritiva, dando-lhe mais liberdade de escolher quais capacitações fazer entre uma seleção disponibilizada pela empresa, dando liberdade à sua força de trabalho para, também, decidir que caminho quer seguir dentro da organização (ALMEIDA, 2013). Portanto, como as trilhas são desenvolvidas alinhadas com a formação por competências, também estão alinhadas com o plano de carreira que seus profissionais podem almejar seguir na organização, indo além de um mero plano de cargos e salários (FREITAS; BRANDÃO, 2006).

Assim, as Trilhas de Aprendizagem dão autonomia e responsabilidade para os empregados sobre seu processo de desenvolvimento de competências, conciliando aspirações do colaborador com os objetivos organizacionais do empregador (FREITAS, 2002; ALMEIDA, 2013). O empregador mostra o caminho e dá as possibilidades, o empregado mostra interesse e se dedica à realização da Trilha de Aprendizagem. Esse processo compartilhado de desenvolvimento dos trabalhadores gera corresponsabilidade que tem demonstrado resultados positivos, porque gera valor para ambas as partes (FREITAS; BRANDÃO, 2006; MULLER, 2009; ALMEIDA, 2013).

Em um Modelo de Gestão baseado em Competências, o desenvolvimento dos trabalhadores da organização deve ser, cuidadosamente, planejado. Por meio da estratégia organizacional, as competências organizacionais essenciais devem ser mapeadas; com estas, as competências profissionais dos trabalhadores devem ser definidas. Neste mapeamento, devem ser observadas as competências, tanto organizacionais, quanto profissionais, que serão necessárias em médio prazo. Perante este diagnóstico, será possível identificar quais competências precisam ser desenvolvidas nos trabalhadores da empresa (desenvolvimento de competências internas) ou captadas de profissionais externos (contratação de novos profissionais efetivos ou temporários). O próximo passo é formular a estratégia de desenvolvimento das competências requeridas (lacuna identificada) e definir os indicadores de desempenho individuais e de grupo que serão monitorados. A última etapa diz respeito ao acompanhamento e à avaliação que devem ser contínuos (GUIMARÃES et al., 2001, BRANDÃO, 2017).

Corroborando Guimarães et al. (2001) e Brandão (2017), após realizada a oferta da Trilha de Aprendizagem, devem-se acompanhar os profissionais formados em sua prática do trabalho, observando seu desempenho e mapeamento novas lacunas de competências, construindo desta forma um acompanhamento consistente e ofertando novas possibilidades de desenvolvimento de competências ou até mesmo mapeando a necessidade de atualização da Trilha de Aprendizagem inicialmente construída (WICH; POLLOCK; JEFFERSON, 2011; SILVA, 2019). 
Considerando que a estratégia de ofertar Trilhas de Aprendizagem como forma de fomentar o desenvolvimento das competências em seus profissionais, quais diretrizes deveriam ser consideradas para facilitar esse processo de construção de uma Trilha de Aprendizagem para profissionais do setor industrial da Sociedade em Transformação Digital? Procurando resposta para este questionamento, buscou-se identificar quais seriam essas diretrizes.

\section{METODOLOGIA}

Buscando mapear quais seriam as diretrizes para construção de Trilhas de Aprendizagem para desenvolvimento de Competências nos profissionais do setor industrial para a Sociedade em Transformação Digital, realizou-se uma pesquisa bibliográfica, com etapas exploratória e utilizando-se da Revisão Sistemática de Literatura (RSL) em bases de dados internacionais, conforme descrito a seguir.

A metodologia utilizada para realizar a revisão bibliográfica nas bases de dados internacionais tem como base o Preferred Reporting Items for Systematic Reviews na Meta-Analyses - PRISMA (MOHER et al., 2009) para a definição das etapas.

Para a definição da estratégia de busca, bem como a seleção das bases de dados mais aderentes ao tema pesquisado, as pesquisadoras contaram com o apoio do Serviço de Referência da Biblioteca Universitária da Universidade Federal de Santa Catarina.

Esta revisão foi realizada mediante definição das palavras-chave da pesquisa, configuradas como: (("fourth industrial revolution" OR "Industry 4.0" OR "Advanced Manufacturing” OR "Smart factory") AND (skill OR Skills OR competence OR competencies OR ability OR capability OR "Professional qualifications" $O R$ education OR training OR "Corporative education" OR "Corporative university" OR "Corporate universities")) em inglês, nas seguintes bases de dados: Scopus, Web of Science (WOF), Eric, Library \& Information Science Abstracts (LISA), Emerald, Compendex e Scielo.
Como se pode observar, não foi utilizado o termo Trilha de Aprendizagem nesta busca, pois identificou-se que um limitado número de estudos trazia esse termo, o que poderia limitar muito o retorno de artigos analisados, dando uma falsa impressão que haveria poucos estudos publicados relacionados ao escopo pesquisado.

Para definição desta seleção de bases de dados, foram considerados a abrangência interdisciplinar ou o foco na área educacional, bem como a variedade de editoras, buscando, com isso, identificar o que havia de estado da arte no assunto desenvolvimento de competências de profissionais da Sociedade em Transformação Digital publicado no meio científico.

Por meio de uma pesquisa exploratória em estudos que tratam da Sociedade em Transformação Digital, foram elencados os seguintes critérios de inclusão e exclusão, adotados nas etapas de análise de elegibilidade das referências. Os artigos que foram incluídos no estudo deveriam reportar: estudos empíricos de desenvolvimento de competências nos profissionais da Sociedade em Transformação Digital; relatar desenvolvimento de competências dos profissionais do setor industrial; estudos publicados a partir do ano 2.000, pois, como se buscavam estudos empíricos, e os autores que estudam a Sociedade em Transformação Digital corroboram que esta Era iniciou no século XXI, possivelmente, não encontraríamos relatos de estudos empíricos antes desta data.

Foram excluídos do estudo os artigos que não tratavam de desenvolvimento de competências nos profissionais do setor industrial da Sociedade em Transformação Digital; tratavam apenas de tecnologias da Sociedade em Transformação Digital; tratavam da Sociedade em Transformação Digital de forma genérica; tratavam de desenvolvimento de competências no âmbito unicamente escolar, sem relação direta com a prática no trabalho; estudos publicados antes do ano 2.000; estudos que não estivessem disponíveis de forma gratuita na internet, acesso livre.

A estratégia de busca foi realizada no dia 24 de outubro de 2018, considerando as especificidades de cada base de dados, conforme apresentado no quadro 1 . 
Quadro 1 - Estratégia de busca utilizada nas bases de dados

\begin{tabular}{|c|c|c|}
\hline Bases de dados selecionadas & Delimitação & $\begin{array}{l}\text { Número de } \\
\text { referências } \\
\text { localizadas }\end{array}$ \\
\hline $\begin{array}{l}\text { SCOPUS (Elsevier) } \\
\text { https://www.scopus.com }\end{array}$ & $\begin{array}{l}\text { Busca avançada por títulos, resumos e palavras- } \\
\text { chave, restringindo por article e review. }\end{array}$ & 768 \\
\hline $\begin{array}{l}\text { Web of Science (Clarivate Analytics / } \\
\text { Thomson Reuters) } \\
\text { http://www.webofknowledge.com/ }\end{array}$ & $\begin{array}{l}\text { Busca avançada por tópico, restringindo por } \\
\text { article e review }\end{array}$ & 497 \\
\hline $\begin{array}{l}\text { Eric (Proquest) } \\
\text { https://search.proquest.com/eric }\end{array}$ & $\begin{array}{l}\text { Busca avançada por título, resumo e } \\
\text { identificador (palavra-chave), restringindo por } \\
\text { article, dissertation and thesis }\end{array}$ & 36 \\
\hline $\begin{array}{l}\text { Library \& Information Science } \\
\text { Abstracts - LISA (Proquest) } \\
\text { https://s earch-proquest.e z } 46 . \\
\text { periodicos.capes.gov.br/lisa }\end{array}$ & $\begin{array}{l}\text { Busca avançada por título e resumo, restringindo } \\
\text { por article. }\end{array}$ & 6 \\
\hline $\begin{array}{l}\text { Emerald } \\
\text { https://www.emeraldinsight.com/ }\end{array}$ & $\begin{array}{l}\text { Busca avançada por título e resumo, restringindo } \\
\text { por article and chapters. }\end{array}$ & 70 \\
\hline $\begin{array}{l}\text { Scielo } \\
\text { http://www.scielo.org/php/index.php }\end{array}$ & $\begin{array}{l}\text { Busca considerando os filtros: todos os índices } \\
\text { e regional. }\end{array}$ & 6 \\
\hline $\begin{array}{l}\text { Compendex (Engineering Village) } \\
\text { https://www.engineeringvillage.com/ } \\
\text { search/quick.url }\end{array}$ & $\begin{array}{l}\text { Busca avançada por título, resumo e palavras- } \\
\text { chave, restringindo apenas journal article. }\end{array}$ & 582 \\
\hline
\end{tabular}

Fonte: elaborado pelas autoras.

As referências elencadas nas fontes primárias foram exportadas para o software Thomson Reuters EndNote (), versão X8, em grupos criados separadamente, por base específica de dados. Na sequência, todas as referências passaram a fazer parte de um único grupo, e as duplicadas foram eliminadas. Do total de referências, as pesquisadoras analisaram os títulos e descartaram os que não apresentavam coerência com os critérios de busca. Em seguida, foi realizada a leitura dos resumos para, finalmente, a leitura por completo das referências selecionadas na amostra.

O processo de elegibilidade das referências foi realizado em quatro etapas, a saber:

$\mathrm{Na}$ etapa 1, com apoio do software EndNote $\mathrm{C}$, versão $\mathrm{X} 8$, as referências duplicadas foram identificadas e removidas. Assim, das 1.965 referências, restaram 1.061 na amostra. Ou seja, foram identificadas 904 referências duplicadas.

$\mathrm{Na}$ etapa 2, foi realizada a leitura dos títulos das 1.061 referências da amostra e iden- tificado que 767 referências tratavam de pesquisas relacionadas a novos materiais ou novos processos de fabricação atrelados à indústria 4.0, especialmente a manufatura aditiva (a impressão 3D) ou sobre performance de produção e produção flexível.

Portanto, à luz dos critérios de inclusão e exclusão definidos para esta revisão bibliográfica, essas referências foram descartadas, restando, na amostra, 294 referências selecionadas para a leitura dos resumos.

$\mathrm{Na}$ etapa 3, foi realizada a leitura dos resumos das 294 referências da amostra, que possibilitou selecionar 82 referências para a leitura completa.

Entre as referências descartadas, por não se encaixarem nos critérios de inclusão definidos, relacionamos 58 artigos que tratavam da Sociedade em Transformação Digital de forma geral; 75 artigos que trataram da necessidade de ajustes nos currículos dos cursos para adequar a formação dos profissionais, especialmente engenheiros; 8 artigos que não tinham resumo na 
sua estrutura; 14 artigos que tratavam da relação entre trabalhadores robôs e humanos, e 57 artigos foram descartados por terem sido publicados antes de 2.000.

$\mathrm{Na}$ etapa 4, entre os 82 artigos selecionados para leitura dos resumos, aplicou-se seu critério de localização de forma gratuita na internet (acesso livre), restando 52 artigos que foram lidos na integra. Nesta análise de leitura completa dos artigos, mais 22 referências foram excluídas da amostra pelos seguintes motivos: 9 por tratar de Indústria 4.0 em geral; 8 por tratar de mudança nos currículos dos cursos; 1 por ser duplicado, 3 por tratarem da relação humanos e robôs, 1 por tratar de competências de profissionais de outra área, que não era o setor industrial. Assim, ao final deste processo sistemático de revisão da literatura, foram consideradas 30 referências na análise realizada.

Nas 4 etapas do processo de análise, quando se ficou em dúvida se a referência se encaixava ou não nos critérios de inclusão e exclusão definidos, eles foram mantidos na amostra, permitindo que uma análise mais detalhada fosse realizada na próxima etapa.

Além desta pesquisa em bases de dados internacionais, foi realizada uma pesquisa bibliográfica e documental exploratória em outras fontes, buscando contribuições de instituições que são referência em formação de profissionais para a indústria ou formação de competências profissionais de modo geral, possibilitando, por meio de uma análise interpretativa (MARCONI; LAKATOS, 2010), a definição de diretrizes para a construção de Trilhas de Aprendizagem, que são apresentadas na próxima seção, e compuseram o universo de fontes consultadas para a identificação das diretrizes buscadas para a construção de Trilhas de Aprendizagem.

Por meio da análise dos cases avaliados na revisão sistemática realizada em artigos científicos empíricos apresentados, foi possível identificar 27 diretrizes para o desenvolvimento de Trilhas de Aprendizagem para desenvolvimento de profissionais do setor industrial da Sociedade em Transformação Digital, que são apresentadas no quadro 2, na ordem numérica de 1 a 27.

A partir da pesquisa bibliográfica e documental exploratória (MARCONI; LAKATOS, 2010), em que foi realizada a análise de algumas metodologias de desenvolvimento de competências, pôde-se identificar mais 5 diretrizes, que, somadas com as 27 identificadas anteriormente, compuseram as 32 diretrizes para a construção de uma Trilha de Aprendizagem para o desenvolvimento de Competências dos profissionais do setor industrial da Sociedade em Transformação Digital, apresentadas no quadro 2. As cinco diretrizes adicionadas estão apresentadas no quadro 2 , na ordem numérica de 28 a 32.

Por analisar um problema em um contexto, a necessidade de se ter diretrizes para construção de uma Trilha de Aprendizagem em um cenário específico, esta pesquisa pode ser caracterizada como propositiva (ALVES-MAZZOTTI, 2001; SERRA, 2006), utilizando-se de métodos científicos para desenvolver um artefato (BUNGE, 1985), a Matriz de Diretrizes proposta.

Para definir este conjunto de diretrizes, bem como a categorização delas em grupos, utilizou-se a metodologia prescrita por Bardin (2011), que consiste em três passos de análise, sendo eles: pré-análise, exploração e tratamento dos materiais. Surgindo a Matriz contendo 32 diretrizes (quadro 2), categorizadas em 3 grupos (figura 1) que são apresentadas em detalhes na seção a seguir.

\section{RESULTADOS}

Aplicando-se o percurso metodológico descrito, buscando facilitar a construção de Trilhas de Aprendizagem, identificou-se um conjunto de 32 (trinta e duas) diretrizes para construção de Trilhas de Aprendizagem para profissionais da Sociedade em Transformação Digital (quadro 2) que, após analisadas, foram categorizadas em três grupos, sendo: Construção da Trilha, Execução da Trilha e Transferência para a Prática do Trabalho (figura 1). 
No quadro 2, estão relacionadas a esta matriz, contendo as 32 (trinta e duas) diretrizes; e, na coluna denominada 'Diretrizes', é apresentada a nominação de cada uma delas. Esta nomeação foi realizada por meio da descrição que está apresentada na coluna seguinte, denominada 'Descrição'. Por fim, na coluna denominada 'Autores', está relacionado a partir dos conteúdos de quais autores foi possível fazer essa categorização (BARDIN, 2011).

A nomeação das diretrizes apresentada na primeira coluna, bem como a descrição de cada diretriz apresentada na segunda coluna foram elaboradas pelas autoras por meio da análise interpretativa dos conteúdos dos autores relacionados na terceira coluna (BARDIN, 2011).

Quadro 2 - Matriz de Diretrizes para a construção de Trilhas de Aprendizagem a profissionais do setor industrial da Sociedade em Transformação Digital

\begin{tabular}{|c|c|c|}
\hline Diretrizes & Descrição & Autores \\
\hline 1 - Diagnóstico & $\begin{array}{l}\text { Identificar lacunas de competência e } \\
\text { competências críticas que precisam } \\
\text { ser desenvolvidas por grupo de } \\
\text { profissionais. }\end{array}$ & $\begin{array}{l}\text { (PAGELL; HANDFIELD; BARBER, } \\
\text { 2000; PENNATHUR; MITAL, } \\
\text { 2003; DONOVAN; MARITZ; } \\
\text { MCLELLAN, 2013; NAGAR; RAJ, } \\
\text { 2013; KINKEL; SCHEMMANN, } \\
\text { LICHTNER, 2017; ANTOSZ, 2018; } \\
\text { WICH; POLLOCK; JEFFERSON, } \\
\text { 2011; ALVES, 2016) }\end{array}$ \\
\hline 2 - Mapeamento & $\begin{array}{l}\text { Mapear as competências que } \\
\text { precisam ser desenvolvidas com } \\
\text { base nas tarefas realizadas pelos } \\
\text { profissionais. }\end{array}$ & $\begin{array}{l}\text { (PENNATHUR; MITAL, 2003; } \\
\text { ALVES, 2016; SENAI, 2019) }\end{array}$ \\
\hline $\begin{array}{l}3 \text { - Stakeholders } \\
\text { internos }\end{array}$ & $\begin{array}{l}\text { Envolver os gestores de RH com } \\
\text { os gestores das áreas técnicas na } \\
\text { definição do escopo dos treinamentos. }\end{array}$ & (WALDECK; LEFFAKIS, 2006) \\
\hline $\begin{array}{l}4 \text { - Competências } \\
\text { Socioemocionais }\end{array}$ & $\begin{array}{l}\text { Desenvolver competências } \\
\text { socioemocionais. }\end{array}$ & $\begin{array}{l}\text { (BORGES; TAN, 2017; KÜSTERS; } \\
\text { PRAB; GLOY, 2017; PERINI et al., } \\
\text { 2017; PIÑOL et al., 2017; KOLB, } \\
\text { 1971, 1984; SENAI, 2019; FREIRE } \\
\text { et al., 2019) }\end{array}$ \\
\hline $\begin{array}{l}5 \text { - Competências } \\
\text { Gerenciais }\end{array}$ & Desenvolver competências gerenciais. & $\begin{array}{l}\text { (KÜSTERS; PRAB; GLOY, 2017; } \\
\text { PERINI et al., 2017; PIÑOL et } \\
\text { al., 2017; CORDERO; WALSH; } \\
\text { KIRCHHOFF, 2009; FREIRE et al. } \\
\text { 2016a; FREIRE et al., 2019) }\end{array}$ \\
\hline $\begin{array}{l}6 \text { - Competências } \\
\text { STEAM }\end{array}$ & $\begin{array}{l}\text { Desenvolver competências } \\
\text { multidisciplinares, como as } \\
\text { competências STEAM. }\end{array}$ & $\begin{array}{l}\text { (KOLB, 1971, 1984; FREIRE et al., } \\
\text { 2016a; FREIRE et al., 2019) }\end{array}$ \\
\hline $\begin{array}{l}7 \text { - Competências } \\
\text { Técnicas }\end{array}$ & $\begin{array}{l}\text { Desenvolver conhecimentos, } \\
\text { habilidades e atitudes relacionadas às } \\
\text { competências profissionais - a função } \\
\text { laboral. }\end{array}$ & (SENAI, 2019) \\
\hline 8 - Tecnologias & $\begin{array}{l}\text { Relacionar as competências } \\
\text { desenvolvidas com as tecnologias } \\
\text { habilitadoras da Sociedade em } \\
\text { Transformação Digital. }\end{array}$ & $\begin{array}{l}\text { (KÜSTERS; PRAB; GLOY, } \\
\text { 2017; PIÑOL et al., 2017; BAYO- } \\
\text { MORIONES; BILLON; LERA- } \\
\text { LOPEZ, 2008) }\end{array}$ \\
\hline
\end{tabular}




\begin{tabular}{|c|c|c|}
\hline 9 - Currículo & $\begin{array}{l}\text { Elaborar os currículos das } \\
\text { ofertas formativas, partindo das } \\
\text { competências críticas que precisam } \\
\text { ser desenvolvidas. }\end{array}$ & $\begin{array}{l}\text { (KINKEL; SCHEMMANN, } \\
\text { LICHTNER, 2017; ALVES, 2016) }\end{array}$ \\
\hline 10 - Institucionalização & $\begin{array}{l}\text { Relacionar o objetivo da capacitação } \\
\text { com os objetivos organizacionais } \\
\text { e listar os resultados esperados, } \\
\text { promovendo o engajamento dos } \\
\text { profissionais na participação das } \\
\text { ofertas formativas. }\end{array}$ & $\begin{array}{l}\text { (GURTOO; TRIPATHY, 2001; } \\
\text { DIÉGUEZ CASTRILLÓN; } \\
\text { CANTORNA, 2005; BELLO- } \\
\text { PINTADO, 2015; GORECKY; } \\
\text { MOHAMED MURA, 2017; PIÑOL } \\
\text { et al., 2017; WICH; POLLOCK; } \\
\text { JEFFERSON, 2011; ALVES, 2016; } \\
\text { FREIRE et al., 2016a) }\end{array}$ \\
\hline $\begin{array}{l}11 \text { - Jornada } \\
\text { Formativa }\end{array}$ & $\begin{array}{l}\text { Desenhar a experiência completa } \\
\text { de desenvolvimento profissional } \\
\text { como um processo, desde o convite } \\
\text { da capacitação até o alcance de } \\
\text { resultados superiores na prática } \\
\text { do trabalho, relacionando com os } \\
\text { objetivos organizacionais. }\end{array}$ & $\begin{array}{l}\text { (WICH; POLLOCK; JEFFERSON, } \\
\text { 2011; ALVES, 2016; FREIRE et al., } \\
\text { 2016a) }\end{array}$ \\
\hline $\begin{array}{l}12 \text { - Transferência do } \\
\text { aprendizado }\end{array}$ & $\begin{array}{l}\text { Prever e mesurar a transferência } \\
\text { do aprendizado para a prática } \\
\text { do trabalho, considerando o } \\
\text { acompanhamento que será realizado } \\
\text { pós-capacitação, com suporte } \\
\text { e feedback do líder imediato, } \\
\text { valorizando o desenvolvimento de } \\
\text { prática do trabalho inovadoras. }\end{array}$ & $\begin{array}{l}\text { (PENNATHUR; MITAL, 2003; } \\
\text { SONG et al., 2006; BAYO- } \\
\text { MORIONES; BILLON; LERA- } \\
\text { LOPEZ, 2008; DONOVAN; } \\
\text { MARITZ; MCLELLAN, 2013; } \\
\text { JOVANOVIC; HARTMAN, } \\
\text { 2013; KINKEL; SCHEMMANN, } \\
\text { LICHTNER, 2017; KÜSTERS; } \\
\text { PRAB; GLOY, 2017; LONGO; } \\
\text { NICOLETTI; PADOVANO, 2017; } \\
\text { PENESIS et al., 2017; WICH; } \\
\text { POLLOCK; JEFFERSON, 2011; } \\
\text { ALVES, 2016; KOLB, 1971, 1984; } \\
\text { SENAI, 2019) }\end{array}$ \\
\hline $\begin{array}{l}13 \text { - Perfil de } \\
\text { aprendizado }\end{array}$ & $\begin{array}{l}\text { Desenvolver as soluções } \\
\text { educacionais, observando as formas } \\
\text { com que os profissionais aprendem } \\
\text { mais facilmente. }\end{array}$ & $\begin{array}{l}\text { (PENNATHUR; MITAL, 2003, } \\
\text { ALVES, 2016; KOLB, 1971, 1984; } \\
\text { FREIRE et al., 2019) }\end{array}$ \\
\hline 14 - Adaptabilidade & $\begin{array}{l}\text { Adaptar as ofertas formativas às } \\
\text { necessidades dos estudantes, usando } \\
\text { tecnologias educacionais e estratégias } \\
\text { de ensino variadas para facilitar o } \\
\text { aprendizado. }\end{array}$ & $\begin{array}{l}\text { (JOVANOVIC; HARTMAN, 2013; } \\
\text { GORECKY; MOHAMED MURA, } \\
\text { 2017; KÜSTERS; PRAB; GLOY, } \\
\text { 2017; LONGO; NICOLETTI; } \\
\text { PADOVANO, 2017; PENESIs et al., } \\
\text { 2017; PERINI et al., 2017; WICH; } \\
\text { POLLOCK; JEFFERSON, 2011; } \\
\text { PERUZZINI; PELLICCIARI, 2017; } \\
\text { ALVES, 2016; KOLB, 1971, 1984; } \\
\text { SENAI, 2019; FREIRE et al., 2016a) }\end{array}$ \\
\hline 15 - Acessibilidade & $\begin{array}{l}\text { Prever acesso aos conteúdos de } \\
\text { multiplataformas. }\end{array}$ & (JOVANOVIC; HARTMAN, 2013) \\
\hline
\end{tabular}




\begin{tabular}{|c|c|c|}
\hline 16 - Flexibilização & $\begin{array}{l}\text { Planejar um plano de capacitação } \\
\text { flexível, incluindo educação on-line } \\
\text { e possibilidade de aproveitamento de } \\
\text { estudos. }\end{array}$ & $\begin{array}{l}\text { (PENNATHUR; MITAL, 2003; } \\
\text { JOVANOVIC; HARTMAN, 2013; } \\
\text { ANTOSZ, 2018; SENAI, 2019) }\end{array}$ \\
\hline $\begin{array}{l}17 \text { - Atividades } \\
\text { experienciais }\end{array}$ & $\begin{array}{l}\text { Desenvolver atividades experienciais } \\
\text { (práticas), integrando teoria e prática. }\end{array}$ & $\begin{array}{l}\text { (KARRE et al., 2017; BAYO- } \\
\text { MORIONES; BILLON; LERA- } \\
\text { LOPEZ, 2008; KÜSTERS; PRAB; } \\
\text { GLOY, 2017; PENESIS et al., } \\
\text { 2017; PERINI et al., 2017; PIÑOL } \\
\text { et al., 2017; KOLB, 1971, 1984; } \\
\text { SENAI, 2019; FREIRE et al., 2019; } \\
\text { PENNATHUR; MITAL, 2003; } \\
\text { WICH; POLLOCK; JEFFERSON, } \\
\text { 2011; ALVES, 2016) }\end{array}$ \\
\hline 18 - Performance & Formar equipes de alto desempenho. & $\begin{array}{l}\text { (CORDERO; WALSH; } \\
\text { KIRCHHOFF, 2009; HAN; LIAO, } \\
\text { 2010) }\end{array}$ \\
\hline 19 - Cocriação & $\begin{array}{l}\text { Estimular o aprendizado por meio } \\
\text { de grupos colaborativos de equipes } \\
\text { multidisciplinares. }\end{array}$ & $\begin{array}{l}\text { (PAGELL; HANDFIELD; BARBER, } \\
\text { 2000) }\end{array}$ \\
\hline 20 - Cursos curtos & $\begin{array}{l}\text { Priorizar módulos/cursos com carga } \\
\text { horária curta. }\end{array}$ & $\begin{array}{l}\text { (JOVANOVIC; HARTMAN, 2013; } \\
\text { PIÑOL et al., 2017; ALVES, 2016) }\end{array}$ \\
\hline $\begin{array}{l}21 \text { - Stakeholders } \\
\text { externos }\end{array}$ & $\begin{array}{l}\text { Validar os currículos de ofertas } \\
\text { formativas com empregadores, } \\
\text { relacionando as competências que } \\
\text { precisam ser desenvolvidas, incluindo } \\
\text { a validação de quais tecnologias serão } \\
\text { priorizadas na formação. }\end{array}$ & $\begin{array}{l}\text { (JOVANOVIC; HARTMAN, 2013; } \\
\text { KARRE et al., 2017; PIÑOL et al., } \\
\text { 2017; SENAI, 2019) }\end{array}$ \\
\hline 22 - Inclusão & $\begin{array}{l}\text { Contemplar soluções educacionais } \\
\text { para profissionais com todos os níveis } \\
\text { de instrução. }\end{array}$ & $\begin{array}{l}\text { (DIÉGUEZ CASTRILLÓN; } \\
\text { CANTORNA, 2005; BAYO- } \\
\text { MORIONES; BILLON; LERA- } \\
\text { LOPEZ, 2008; FREIRE et al., 2016a) }\end{array}$ \\
\hline 23 - Extensão & $\begin{array}{l}\text { Estender o plano de qualificação } \\
\text { da força de trabalho para os } \\
\text { stakeholders. }\end{array}$ & $\begin{array}{l}\text { (PENNATHUR; MITAL, 2003; } \\
\text { FREIRE et al., 2016a) }\end{array}$ \\
\hline $\begin{array}{l}24 \text { - Ambiente } \\
\text { organizacional }\end{array}$ & $\begin{array}{l}\text { Priorizar a realização dos } \\
\text { cursos dentro da infraestrutura } \\
\text { organizacional. }\end{array}$ & $\begin{array}{l}\text { (JOVANOVIC; HARTMAN, 2013; } \\
\text { NAGAR; RAJ, 2013; BELLO- } \\
\text { PINTADO, 2015) }\end{array}$ \\
\hline 25 - Priorização & $\begin{array}{l}\text { Desenvolver soluções educacionais } \\
\text { que atinjam o maior número de } \\
\text { profissionais. }\end{array}$ & (NAGAR; RAJ, 2013) \\
\hline 26 - Compartilhamento & $\begin{array}{l}\text { Promover atividades de } \\
\text { compartilhamento do conhecimento } \\
\text { além das ofertas formativas formais } \\
\text { (atividades além dos currículos). Ex. } \\
\text { Comunidade de Prática. }\end{array}$ & $\begin{array}{l}\text { (KINKEL; SCHEMMANN, } \\
\text { LICHTNER, 2017) }\end{array}$ \\
\hline
\end{tabular}




\begin{tabular}{|c|c|c|}
\hline $\begin{array}{l}27 \text { - Gestão do } \\
\text { Conhecimento }\end{array}$ & $\begin{array}{l}\text { Prever estratégias e soluções } \\
\text { para gestão dos conhecimentos } \\
\text { individuais e organizacionais, } \\
\text { para captação, armazenamento, } \\
\text { compartilhamento e cocriação dos } \\
\text { conhecimentos gerados pela rede } \\
\text { organizacional (interorganizacional e } \\
\text { intraorganizacional). }\end{array}$ & (FREIRE et al., 2016a) \\
\hline $\begin{array}{l}28 \text { - Ambientes } \\
\text { cativantes }\end{array}$ & $\begin{array}{l}\text { Prever o desenvolvimento das ofertas } \\
\text { formativas em ambientes cativantes. }\end{array}$ & (FREIRE et al., 2019) \\
\hline $\begin{array}{l}29 \text { - Aprender a } \\
\text { aprender }\end{array}$ & Valorizar o aprender a aprender. & (SENAI, 2019; FREIRE et al., 2019) \\
\hline 30 - Interdisciplinaridade & $\begin{array}{l}\text { Planejar as ofertas formativas } \\
\text { prevendo a interdisciplinaridade. }\end{array}$ & $\begin{array}{l}\text { (PERINI et al., 2017; FREIRE et al., } \\
\text { 2016a) }\end{array}$ \\
\hline 31 - Personalização & $\begin{array}{l}\text { Prever a possibilidade de } \\
\text { personalização das ofertas formativas } \\
\text { de acordo com a necessidade de } \\
\text { desenvolvimento de competências } \\
\text { específicas de grupos de profissionais. }\end{array}$ & $\begin{array}{l}\text { (ALVES, 2016; FREIRE et al., } \\
\text { 2016a) }\end{array}$ \\
\hline $\begin{array}{l}\text { 32- Evolução } \\
\text { customizada }\end{array}$ & $\begin{array}{l}\text { Prever a possibilidade de construção } \\
\text { de novas trilhas ou personalização } \\
\text { de trilhas já desenvolvidas de forma } \\
\text { ágil, atendendo à necessidade } \\
\text { de desenvolvimento de novas } \\
\text { competências cada vez mais acelerada }\end{array}$ & (ALVES, 2016) \\
\hline
\end{tabular}

Fonte: elaborado pelas autoras.

Conforme mencionado anteriormente, por meio da análise interpretativa das publicações selecionadas (BARDIN, 2011), foi possível categorizar as 32 diretrizes em três grandes grupos de Diretrizes para realização de Trilhas de Aprendizagem (figura 1).

Figura 1 - Pirâmide de Diretrizes para a realização Trilha de Aprendizagem para a Sociedade em Transformação Digital

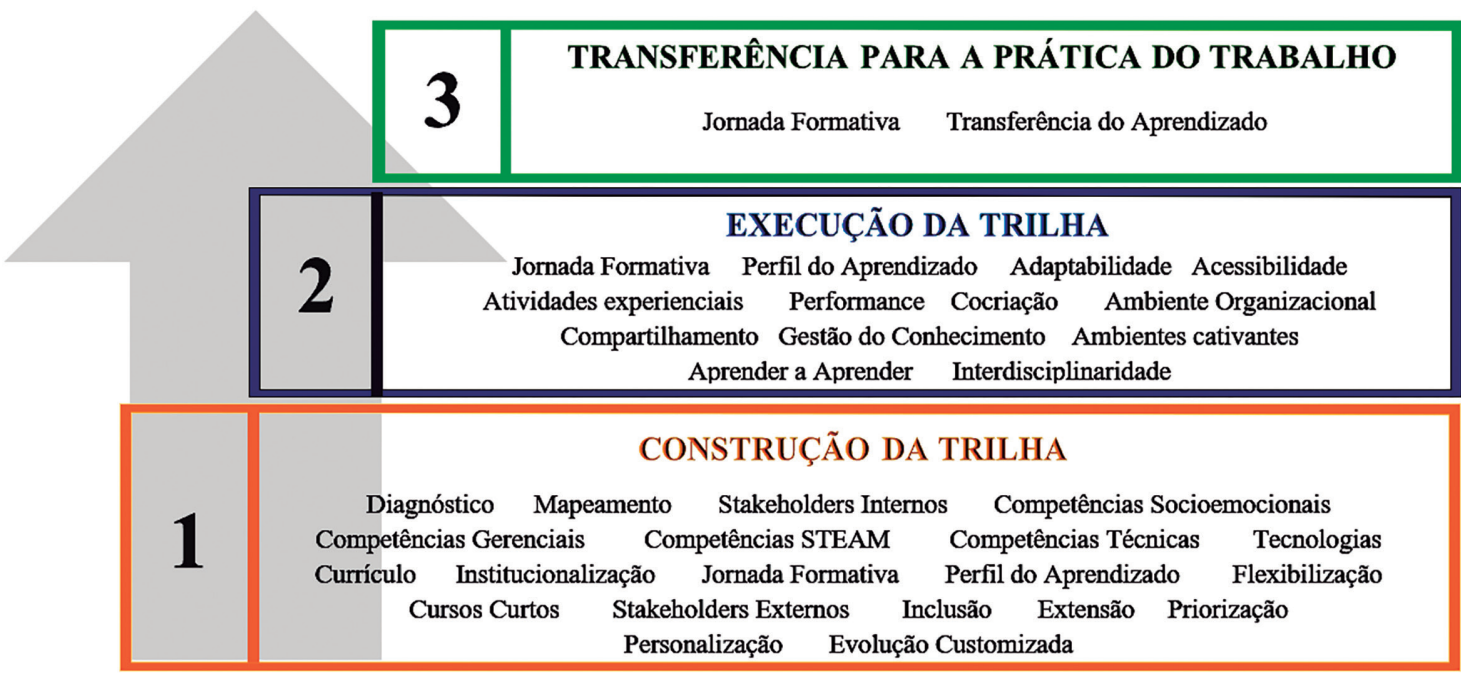

Fonte: elaborada pelas autoras. 
As diretrizes incluídas no grupo denominado Construção da Trilha, que figuram na base da pirâmide, são aquelas que devem ser observadas na etapa de planejamento da oferta da Trilha, etapa que ocorre antes da realização das ofertas formativas; as diretrizes incluídas no grupo denominado Execução da Trilha, que estão no meio da pirâmide, são aquelas que devem ser consideradas no momento de realização das ofertas formativas planejadas.

As diretrizes incluídas no grupo denominado Transferência para a Prática do Trabalho, elencadas no topo da pirâmide, são aquelas que devem ser consideradas na etapa que vêm após a realização da oferta formativa, que é o acompanhamento dos profissionais desenvolvidos visando identificar se eles estão apresentando um desempenho competente em face das competências que foram, ou deveriam ter sido desenvolvidas na formação oferecida. Nesta última etapa, espera-se que o profissional esteja demonstrando um desempenho superior ao observado antes de realizar a Trilha de Aprendizagem (WICH; POLLOCK; JEFFERSON, 2011; SILVA, 2019).

Ao analisar as 20 (vinte) competências relacionadas no Grupo Construção da Trilha (figura 1), que estão relacionadas com o objetivo deste estudo que é identificar diretrizes para a construção de Trilhas de Aprendizagem para o desenvolvimento de competências nos profissionais do setor industrial para a Sociedade em Transformação Digital, pode-se confirmar algumas afirmações assinaladas por autores citados na introdução e fundamentação teórica deste estudo.

Verificou-se a convergência das Diretrizes: 1- Diagnóstico e 2 - Mapeamento com a visão de Guimarães et al. (2001) e Brandão (2017), os quais afirmaram que mapear as competências essenciais da organização e as lacunas de competências dos trabalhadores são os primeiros passos que precisam ser considerados no delineamento de Trilhas de Aprendizagem.

As Diretrizes: 3 - Stakeholders internos, 21 - Stakeholders externos e 23 - Extensão convergem com as afirmações de Meister (1999) e Freire et al. (2016b), os quais demonstraram que a evolução da educação corporativa ultrapassa os muros das organizações, envolvendo além de profissionais da empresa, clientes e cadeia de fornecimento, envolvendo esses atores tanto na construção da Trilha de Aprendizagem como na oferta das soluções de aprendizagem em si.

A necessidade de desenvolvimento de competências além das competências técnicas (SENAI, 2019; FREIRE et al., 2019) é confirmada nas Diretrizes 4 - Competências Sociemocionais, 5 - Competências Gerenciais e 6 Competências STEAM.

A necessidade constante de desenvolvimento de novas competências técnicas, alinhadas com as tecnologias utilizadas, é verificada nas Diretrizes 7 - Competências Técnicas e 8 - Tecnologias, confirmando que, conforme o desenvolvimento tecnológico evolui, as competências, especialmente as competências técnicas, exigidas dos profissionais, são atualizadas (AIRES; FREIRE; SOUZA, 2016), exigindo a requalificação dos profissionais (EBOLI, 2004; MEISTER, 1999; FREIRE et al., 2016a).

As Diretrizes: 9 - Currículo, 10 - Institucionalização e 11 Jornada Formativa também estão correlacionadas, pois, conforme descrito por Freitas e Brandão (2006) Almeida (2013) e Brandão (2017), o currículo dos cursos ofertados em uma Trilha de Aprendizagem precisa ser definido por meio das competências críticas ao negócio mapeadas na organização correlacionado com as lacunas de competências identificadas nos profissionais, relacionando esse conjunto de competências a serem desenvolvidas com os objetivos organizacionais da empresa. Tudo isso de modo que faça sentido para o alcance dos objetivos organizacionais, convergindo com o desenvolvimento dos trabalhadores, sendo um resultado ganha-ganha, ganha a empresa em resultados e ganha o trabalhador em desenvolvimento e reconhecimento.

Também se observou convergência com os pressupostos de Alves (2016) que apresenta orientações na construção de trilhas de aprendizagem que preconizam questões relacionadas 
ao perfil dos profissionais a serem desenvolvidos, como o perfil de aprendizado (Diretriz 13 - Perfil de Aprendizado), a flexibilização com possibilidade de aproveitamento de conhecimentos (Diretriz 16 - Flexibilização), a inclusão de profissionais de diferentes níveis de instrução (Diretriz 22 - Inclusão), a oferta de cursos com cargas horárias reduzidas (Diretriz 20 - Cursos curtos), bem como prevenção da personalização de conteúdos para públicos específicos (Diretriz 31 - Personalização).

Tudo isso alinhado com a priorização da formação do maior número de profissionais (Diretriz -25 - Priorização) e a atualização da trilha de aprendizagem de forma ágil (Diretriz 32 - Evolução customizada) possibilitará à organização ter um processo de formação continuada e atualizada de seus profissionais, com uma jornada de formação completa (Diretriz 11 - Jornada Formativa).

\section{CONCLUSÃO}

Ao alcançar o mapeamento da Matriz, contendo as 32 (trinta e duas) diretrizes para construção de Trilhas de Aprendizagem de desenvolvimento de Competências dos profissionais da Sociedade em Transformação Digital do setor industrial (quadro 2), elencando 20 (vinte) no grupo de diretrizes que devem ser observadas na etapa de construção da Trilha (figura 1), deu-se um passo importante na tentativa de materializar o que deve ser observado no planejamento de Trilhas de Aprendizagem sob a ótica do mundo científico, deixando essa contribuição teórica que pode ser aplicada no mundo organizacional.

Esta pesquisa avança das demais identificadas, por reunir um conjunto de Diretrizes que devem ser consideradas na construção de Trilhas de Aprendizagem para profissionais da Sociedade em Transformação Digital do setor industrial, apoiando-se no método científico para definição do conjunto de diretrizes apresentadas.

Ao considerar os critérios da revisão sistemática realizada nos artigos científicos, apenas estudos empíricos publicados em 7 (sete) bases de dados científicas internacionais, adicionando os estudos localizados na pesquisa exploratória, tornam este estudo consistente, analisando o que há de estudo da arte internacionalmente.

Ao categorizar as 32 (trinta e duas) competências mapeadas, este estudo foi além de seu objetivo inicial de apresentar apenas Diretrizes para construção de Trilhas de Aprendizagem, apresentando, também, diretrizes para a execução da Trilha e Transferência para a prática do trabalho (figura 1). Assim, este conjunto de diretrizes é como um mapa que apresenta uma sugestão do que considerar no caminho completo da formação de profissionais, desde o mapeamento das competências essenciais a serem desenvolvidas até a observação na prática do trabalho se o desempenho competente superior está sendo verificado.

Assim, é possível desenvolver outras estratégias de desenvolvimento das competências relacionadas, bem como mapear novas competências a serem desenvolvidas, entendendo que a organização está sempre buscando resultados superiores em um processo de melhoria contínua e desenvolvimento sustentado, Além do mais, ao considerar que o objeto é o desenvolvimento de competências humanas, este é um trabalho que nunca tem fim, sempre há espaço para novos desenvolvimentos em um ambiente econômico competitivo.

Considerando que esta pesquisa residiu no campo teórico, sugere-se sua aplicação em campo visando ao refinamento das diretrizes mapeadas, bem como sua aplicação na construção de trilhas que não se limitam ao desenvolvimento de Competências para a Sociedade em Transformação Digital e para desenvolvimento de competências em profissionais do setor industrial, verificando, especialmente, a aplicação em profissionais que atuam em outros setores econômicos.

Por isso, sugere-se, em trabalhos futuros, que essas diretrizes sejam validadas na construção de Trilhas de Aprendizagem para o desenvolvimento de profissionais de outros setores produtivos, certificando sua aplicação na íntegra ou necessidade de personalização. 
Desenvolver novas competências profissionais é, e provavelmente continuará sendo, por muito tempo, um dos desafios das organizações: encontrar meios de mapear as competências que precisam ser desenvolvidas. Definir o melhor caminho para desenvolver essas competências de forma ágil e consistente passa a ser um diferencial competitivo para as organizações e para os profissionais desenvolvidos, o que é um diferencial de empregabilidade.

Portanto, pode-se afirmar que ter diretrizes para construção e remodelamento de Trilhas de Aprendizagem torna-se um conhecimento crítico organizacional a ser gerenciado e que seguirá em constante evolução.

\section{REFERÊNCIAS}

AIRES, R. W.A. Desenvolvimento de Competências Gerais para a Sociedade em Transformação Digital: uma Trilha de Aprendizagem para profissionais do setor industrial. 2020. Dissertação (Mestrado em Engenharia e Gestão do Conhecimento) - Centro Tecnológico, Universidade Federal de Santa Catarina, Florianópolis, 2020.

AIRES, R. W. A.; KEMPNER-MOREIRA, F. K.; FREIRE, P. S. Indústria 4.0: competências requeridas aos profissionais da quarta revolução industrial. In: CONGRESSO INTERNACIONAL DE CONHECIMENTO E INOVAÇÃO (CIKI), 7., 2017, Foz do Iguaçu. Anais [...]. Foz do Iguaçu: EGC, UFSC, 2017.

AIRES, R. W. A.; FREIRE, P. S.; SOUZA, J. A. Educação Corporativa como ferramenta para estimular a inovação nas organizações: uma revisão de literatura. In: CONGRESSO BRASILEIRO DE GESTÃO DO CONHECIMENTO - KM BRASIL, 13., 2016, São Paulo. Anais [...]. São Paulo: SBGC, 2016.

ALLES, Martha Alicia. Desarrollo del talento humano: baseado en competencias. Buenos Aires: Granica, 2007.
ALMEIDA, M. A. Trilhas de Aprendizagem: um estudo de caso. In: CONGRESSO NACIONAL DE EXCELÊNCIA EM GESTÃO, 9., 2013, Rio de Janeiro. Anais [...]. Rio de Janeiro, 2013.

ALVES, F. Design de Aprendizagem com uso de canvas: trahentem. São Paulo: DVS Editora, 2016.

ALVES-MAZZOTTI, A. J. Relevância e aplicabilidade da pesquisa em educação. Cadernos de Pesquisa, São Paulo, n. 113, p. 39-50, jul. 2001.

ANTONELLO, Claudia Simone; FLACH Leonardo. Organizações culturais e a aprendizagem baseada em práticas. Cadernos EBAPE. BR, Rio de Janeiro, v. 9, n. 1, p. 156-176, mar. 2011.

ANTONELlO, Cláudia Simone. Aprendizagem na ação revisitada e seu papel no desenvolvimento de competências. Aletheia, n. 26, p. 146-167, jul./dez, 2007. Disponível em: http://www.ulbra.br/upload/9d989a5c700528c00130adf79d7aec94.pdf . Acesso em: 30 out. 2019

ANTOSZ, K. Maintenance - Identification and analysis of the competency gap. Eksploatacja i Niezawodnosc - Maintenance and Reliability, v. 20, n. 3, p. 484-494, 2018. http://dx.doi. org/10.17531/ein.2018.3.19

BARDIN, L. Análise de conteúdo. São Paulo: Edições 70, 2011.

BAYO-MORIONES, A.; BILLON, M.; LERA-LOPEZ, F. Skills, technology and organisational innovation in Spanish firms. International Journal of Manpower, v. 29 , n. 2 , p. 122-145, 2008. https://doi. org/10.1108/01437720810872695

BELLO-PINTADO, A. Bundles of HRM practices and performance: empirical evidence from a Latin American context. Human Resource Management Journal, v. 25, n. 3, p. 311-330, 2015. https://doi.org/10.1111/17488583.12067 
BORGES, L. A.; TAN, K. H. Incorporating human factors into the AAMT selection: a framework and process. International Journal of Production Research, v. 55, n. 5, p. 14591470, 2017. https://doi.org/10.1080/00207543 .2016 .1259668

BRANDÃO, Hugo Pena. Mapeamento de competências: ferramentas, exercícios e aplicações em gestão de pessoas. 2. ed. São Paulo: Atlas, 2017.

BRASIL. Ministério da Educação. Lei $\mathbf{n}^{\circ}$ 9.394, DE 20 DE DEZEMBRO DE 1996. Estabelece as diretrizes e bases da educação nacional. Brasília, DF: Presidência da República, 1996. Disponível em: http://www.planalto.gov. br/ccivil 03/leis/19394.htm/. Acesso em: 30 abr. 2018.

BRASIL. Ministério da Educação (MEC). Base nacional Comum Curricular: educação é a base. Brasília: MEC, 2018.

BRASIL. Ministério da Educação (MEC). Referenciais Curriculares Nacionais da Educação Profissional de Nível Técnico (RCNEP). Brasília: MEC, 2000.

BUNGE, M. Seudociência e Ideologia. Madrid: Alianza, 1985.

CONFEDERAÇÃO NACIONAL DA INDÚSTRIA - CNI. História do SENAI. Brasília, DF, 2016. Disponível em: http://www.portaldaindustria.com.br/senai/institucional/historia/. Acesso em: 30 jul. 2019.

CORDÃO, F. A.; MORAES, F. Educação Profissional no Brasil: síntese histórica e perspectivas. São Paulo: Editora SENAC, 2017.

CORDERO, Rene; WALSH, Steven T.; KIRCHHOFF, Bruce A. Organization technologies, AMT and competent workers: Exploring relationships with manufacturing performance. Journal of Manufacturing Technology Management, v. 20, n. 3, p. 298-313, 2009. https:// doi.org/10.1108/17410380910936765
COSTA, M.; STEFANO, F. A era das fábricas inteligentes está começando. Exame, 2014. Disponível em: https://exame.abril.com.br/revista-exame/a-fabrica-do-futuro/. Acesso em: 30 abr. 2018.

DAVENPORT, T. H.; PRUSAK, L. Conhecimento empresarial: como as organizações gerenciam o seu capital intelectual. Rio de Janeiro: Campus, 1998.

DONOVAN, J. D.; MARITZ, A.; MCLELLAN, A. Innovation training within the Australian advanced manufacturing industry. Journal of Vocational Education \& Training, v. 65, n. 2, p. 256-276, 2013. https://doi.org/10.1080/136368 20.2013.783614

DELUIZ, N. O Modelo das Competências no Mundo do Trabalho e na educação: implicações para o currículo. Boletim Técnico SENAC, 2001. Disponível em: http://www.senac.br/BTS/273/ boltec273b.html. Acesso em: 30 ago. 2018 .

DIÉGUEZ CASTRILLÓN, I.; SINDE CANTORNA, A. I. The effect of the implementation of advanced manufacturing technologies on training in the manufacturing sector. Journal of European Industrial Training, v. 29, n. 4, p. 268-280, 2005. https://doi. org/10.1108/03090590510597124

EBOLI, M. Educação Corporativa no Brasil: mitos e verdades. São Paulo: Gente, 2004.

FREIRE, P. S. et al. Universidade Corporativa em Rede: Considerações Iniciais para um Novo Modelo de Educação Corporativa. Revista Espacios, v. 37, n. 5, 2016a.

FREIRE, P. S. et al. Processo de implantação da Universidade Corporativa em Rede (UCR). Revista Espacios, v. 37, n. 23, 2016 b.

FREIRE, P. S. et al. As quatro dimensões do Método Alexandria para instanciação da neoaprendizagem na prática do ensino superior. In: FREIRE, P. S.; SILVA, T. C.; BRESOLIN, G. G. Universidade Corporativa em Rede: diretrizes iniciais do modelo. Curitiba: CRV, 2019. 
FREITAS, I. A.; BRANDÃO, H. P. Trilhas de aprendizagem como estratégia de TD\&E. In: BORGES-ANDRADE, J. E.; ABBAD, G.; MOURÃO, L. (org.). Treinamento, Desenvolvimento e Educação em Organizações e Trabalho: fundamentos para a gestão de pessoas. Porto Alegre (RS): Artmed, 2006. p. 97-113.

FREITAS, I. A. Trilhas de desenvolvimento profissional: da teoria a prática. In: ENANPAD, 26., 2002, Salvador. Anais [...]. Salvador: ANPAD, 2002.

GORECKY, D.; KHAMIS, M.; MURA, K. Introduction and Establishment of Virtual Training in the Factory of the Future. International Journal of Computer Integrated Manufacturing, v. 30, n. 1, p. 182-190, 2017. https:// doi.org/10.1080/0951192X.2015.1067918

GUI, R. T. Trilhas de desenvolvimento professional: plano de trabalho. Brasília: Mimeo, 2000 .

GUIMARÃES, T. A. et al. Forecasting core competencies in na $R \& D$ environment. R\&D Management Review, Manchester, UK, v. 31, n. 3, p. 249-255, 2001.

GURTOO, A.; TRIPATHY, A. Workers' orientation towards advanced manufacturing technology: a study of the Indian automobile industry. International Journal of Human Resources Development and Management, v. 1, n. 2/3/4, 2001. https://doi.org/10.1504/ IJHRDM.2001.001012

HARARI, Yuval Noah. Sapiens: uma breve história da humanidade. 38. ed. Porto Alegre: L\&PM, 2018.

HAN, T. S.; LIAO, W. C. Computer-integrated manufacturing and high performance work system: The case of Taiwan. The International Journal of Human Resource Management, v. 21, n. 3, p. 434-454, 2010. https://doi. org/10.1080/09585190903549114

JOVANOVIC, V.; HARTMAN, N. W. Webbased virtual learning for digital manufacturing fundamentals for automotive workforce training. Engineering Technology Faculty Publications, v. 23 , n. $3-4$, p. $300-310,2013$. DOI: 10.1504/ijceell.2013.055403

JUNQUEIRA, C. B. Trilhas de desenvolvimento profissional. Porto Alegre: Mimeo, 2000.

KARRE, H. et al. Transition towards an Industry 4.0 State of the LeanLab at Graz University of Technology. Procedia Manufacturing, v. 9 p. 206-2013, 2017. https://doi.org/10.1016/j. promfg.2017.04.006

KINKEL, S.; SCHEMMANN, B.; LICHTNER, R. Critical competencies for the innovativeness of value creation champions: identifying challenges and work-integrated solutions. Procedia Manufacturing, v. 9, p. 323-330, 2017. https:// doi.org/10.1016/j.promfg.2017.04.021

KOLB, D. A. Individual learning styles and the learning process. Massachusetts: Sloan School of Management. 1971.

KOLB, D. A. Experiential learning: experience as the source of learning and development. New Jersey: Prentice Hall, 1984.

KÜSTERS, D.; PRAB, N.; GLOY, Y. S. Textile Learning Factory 4.0 - Preparing Germany's Textile Industry for the Digital Future. Procedia Manufacturing, v. 9, p. 214-221, 2017. https://doi.org/10.1016/j.promfg.2017.04.035

LE BOTERF, G. Competénce et navigation professionnelle. Paris: éditions D'Organisacion, 1999.

LONGO, F.; NICOLETTI, L.; PADOVANO, A. Smart operators in industry 4.0: A human-centered approach to enhance operators' capabilities and competencies within the new smart factory context. Computers \& industrial Engineering, v. 113, p. 144-159, 2017. https://doi.org/10.1016/j.cie.2017.09.016

MARCONI, M. A.; LAKATOS, E. M. Fundamentos de Metodologia Científica. 7. ed. São Paulo: Atlas, 2010. 
MCKINSEY. Industry $\mathbf{4 . 0}$ at McKinsey's model factories: get ready for the disruptive wave. [S.l.: s.n.], 2016.

MEISTER, J. C. Educação Corporativa: a gestão do capital intelectual através das Universidades Corporativas. São Paulo: Pearson Books, 1999.

MOHER, D. et al. Preferred reporting items for systematic reviews and meta-analyses: the PRISMA Statement. Open Medicine, v. 3, n. 3, p. 123-130, 2009.

MULLER, Claudia Cristina. Educação a Distância nas Organizações. Curitiba: IESDE Brasil S.A., 2009. v. 1.

NAGAR, B.; RAJ, T. An analytical case study of an advanced manufacturing system for evaluating the impact of human enablers in its performance. Journal of Advances in Management Research, v. 10, n. 1, p. 85-99, 2013. https://doi.org/10.1108/09727981311327785

PAGELL, M.; HANDFIELD, R. B.; BARBER, A. E. Effects of operational employee skills on advanced manufacturing technology performance. Production and Operations Management, v. 9, n. 3, p. 222-238, 2000. https://doi. org/10.1111/j.1937-5956.2000.tb00135.x

PENNATHUR, A.; MITAL, A. Worker mobility and training in advanced manufacturing. International Journal of industrial Ergonomics, v. 32, n. 6, p. 363-388, 2003. https://doi. org/10.1016/S0169-8141(03)00076-3

PENESIS, I. et al. Reskilling the manufacturing workforce and developing capabilities for the future. Australasian Journal of Engineering Education, v. 22, n. 1, p. 14-22, 2017. DOI: 10.1080/22054952.2017.1338228 Disponível em: http://dx.doi.org/10.1080/22054952.2017. 1338228 Acesso em: 30 out. 2018.

PERRENOUD, P. Avaliação: da excelência à regulação das aprendizagens: entre duas lógicas. Porto Alegre: Artmed, 1999.
PERINI, S. et al. Training advanced skills for sustainable manufacturing: a digital serious game. Procedia Manufacturing, v. 11, p. 1536-15-43, 2017. https://doi.org/10.1016/j. promfg.2017.07.286

PERUZZINI, M.; PELLICCIARI, M. A framework to design a human-centred adaptive manufacturing system for aging workers. Advanced Engineering Informatics, v. 33, p. 330-349, 2017. https://doi.org/10.1016/j. aei.2017.02.003

PIÑOL, T. C.; PORTA, S. A.; ARÉVALO, M. C. R. Minguella-Canela, J. Study of the training needs of industrial companies in the Barcelona Area and proposal of Training Courses and Methodologies to enhance further competitiveness. Procedia Manufacturing, v. 13, p. 1426-1431, 2017. https://doi.org/10.1016/j. promfg.2017.09.159

SCHWAB, Klaus. A quarta revolução industrial. São Paulo: Edipro, 2016.

SCHWAB, Klaus; DAVIS, Nicholas. Aplicando a quarta revolução industrial. São Paulo: Edipro, 2018.

SENAI - SERVIÇO NACIONAL DE APRENDIZAGEM INDUSTRIAL. Metodologia SENAI de Educação Profissional. Brasília: SENAI/DN, 2019.

SENGE, P. M. A quinta disciplina: arte e prática da organização que aprende. 28. ed. São Paulo: Best Seller, 2012.

SERRA, G. G. Pesquisa em arquitetura e urbanismo: guia prático para o trabalho de pesquisadores em pós-graduação. São Paulo: EdUSP, 2006.

SILVA, T. C. Framework Ponte Tap: gestão da curva de aprendizagem para a efetivação da transferência de aprendizagem para a prática do trabalho. 2019. Tese (Doutorado em Engenharia e Gestão do Conhecimento) Universidade Federal de Santa Catarina, Florianópolis, 2019. 
SLEIGHT, D. A. A developmental history of training in the United States and Europe. [S.l.: s.n.], 1993.

SONG, J. B. et al. Effects of human factors on performance of advanced manufacturing technology: an empirical examination. Materials Science Forum, v. 505-507, p. 901-906, 2006. https://doi.org/10.4028/www.scientific.net/ MSF.505-507.901

STEIL, A. V. Competências e Aprendizagem Organizacional: como planejar programas de capacitação para que as competências individuais auxiliem a organização a aprender. Florianópolis: Stela, 2006.

STEVAN JÚNIOR, S. L.; LEME, M. O.; SANTOS, M. M. D. Indústria 4.0: fundamentos, perspectivas e aplicações. São Paulo: Érica, 2018.

WALDECK, N. E.; LEFFAKIS, Z. M. HR perceptions and the provision of workforce training in an AMT environment: An empirical study. Omega, v. 35, n. 2, p. 161-172, 2006. https://doi.org/10.1016/j.omega.2005.05.001

WICH, C.; POLLOCK, R.; JEFFERSON, A. 6Ds: as seis disciplinas que transformam educação em resultados para o negócio. São Paulo: Évora, 2011.

ZARIFIAN, P. A gestão da e pela competência. In: SEMINÁRIO INTERNACIONAL EDUCAÇÃO PROFISSIONAL, TRABALHO E COMPETÊNCIAS, Rio de Janeiro, 1996. Anais [...]. Rio de Janeiro: Centro Internacional para Educação, Trabalho e Transferência de Tecnologia, 1996.

\section{AGRADECIMENTO}

Ao Fundo de Apoio à Manutenção e ao Desenvolvimento da Educação Superior FUMDES pelo apoio financeiro recebido por meio do Programa UNIEDU/Pós-Graduação para realização desta pesquisa. 